\title{
Anesthesia-Related Complaint Cases at Kermanshah Medical Council Between 1993 and 2011
}

\author{
Mitra Yari ${ }^{1}$, Mojtaba Niazi ${ }^{2}$, Hossein Farzam ${ }^{2}$ and Parisa Golfam ${ }^{1, *}$ \\ ${ }^{1}$ Emam Reza Hospital, Kermanshah University of Medical Sciences, Kermanshah, Iran \\ ${ }^{2}$ Emam Ali Hospital, Kermanshah University of Medical Sciences, Kermanshah, Iran \\ "Corresponding author: Associate Professor of Anesthesiology, Emam Reza Hospital, Kermanshah University of Medical Sciences, Kermanshah, Iran. Tel: +98-9181310864, Email: \\ pgolfam@kums.ac.ir
}

Received 2018 March 04; Revised 2018 December 29; Accepted 2019 January 01.

\begin{abstract}
The analysis of complaints in different fields of medicine helps understand medical malpractices. This study investigated the complaints related to anesthesia services raised in Kermanshah Medical Council. A total of 35 complaints were found, among which were 16 cases of death and eight cases concluded the malpractice of anesthesiologist. In $21 \%$ of cases, the anesthesiologist was found guilty. About half of the complaints and confirmed cases of malpractices pertained to death or permanent brain damage.
\end{abstract}

Keywords: Anesthesia Complaint, Anesthesia Malpractice, Perioperative Death, Anesthesia Complication

\section{Background}

Patient harm caused by medical cares is commonplace, but the majority of harmed patients are reluctant to sue (1).

The majority of cases leading to complaints were about brain damage, death, insufficient anesthesia and awareness, and dental, airway, and peripheral nerve damage. Some studies have compared the complications of general, neuraxial and regional anesthesia (2).

In Iran, a number of cross-sectional studies have been conducted on the above subject, such as a study conducted in Tehran by Mahfouzi and Zamani who investigated cases between 1993 and 2003, and most complaints were about damage leading to death followed by nervous system damage. Most of the damage (90\%) had occurred during general anesthesia, and 5\% during regional anesthesia (3).

Staender et al. investigated complaints between 1987 and 2008 in Switzerland. A total of 171 complaints concerned anesthesia-related damage, of which, 54\% were in regional anesthesia, 28\% in general anesthesia, and 18\% related to procedures during anesthesia. In all these complaints, 12 cases had led to death and 63 cases to permanent damage (4).

Cook et al. investigated complaints filed between 1995 and 2007, and examined complaints about the pattern of airway damage during anesthesia and their financial damage. Of the 841 complaints assessed, 96 cases were about dental damage, 67 about airway damage, and 24 about breathing complaint (2).

In a study conducted in Germany by Hachenberg et al. between 2005 and 2007, the experts' views about anesthesia-related complaints were assessed, and argued that conceptual conclusive statements were observed in only $60 \%$ of experts' reports, and most of their views were unscientific, and recommended development of guidelines for expert reports (5).

In a study by Cranshaw et al., complaint cases associated with anesthesia were examined in Royal Bournemouth Hospital in England from 1995 to 2007. Of the total of 93 complaints, 16 related to taking the wrong medication, 25 cases to the wrong dose of medication, and 9 cases to the overdose of opioids in the neuraxial block (6).

In Saudi Arabia, Samarkandi studied complaints about anesthetic services between 1999 and 2003, and in this fiveyear period, 1765 complaints about the medical staff had been registered, of which, $4 \%$ (76 cases) related to anesthesia (7).

Iran's Medical Council is responsible for dealing with complaints about various medical disciplines. The analysis of anesthesia complaints can reveal the reasons for patient dissatisfaction, medical malpractice, and factors leading to these complaints. Knowledge of medical errors leading to medical negligence can prevent repetition of errors, and in addition to promoting the patients' health, reduce financial damage, emotional complications, and profes- 
sional damage to doctors. In absence of comprehensive information about complications of anesthesia and related complaints in the country, we felt the need that reviewing complaints about anesthetic services and collecting this information from different provinces can lead to practical results.

\section{Objectives}

The present study was designed and conducted based on this objective.

\section{Methods}

After obtaining permission from the Research Council and Deputy Research and Technology of Kermanshah University of Medical Sciences, and the Ethics Committee of Imam Reza Clinical Research Development Unit, and coordination with the Medical Council of Kermanshah province, first, all complaint cases were reviewed to extract anesthesia complaints. The complaints issued with a verdict of stay of proceedings were excluded from the study. Then, the information checklist was completed. However, some cases did not have all the data. Next, data collected were analyzed in SPSS-16 using tables of frequency and statistical indices.

\section{Results}

Of the complaint cases relating to medical team between 1993 and 2011, 35 complaints were about anesthetic services, of which, 18 complaints (51.4\%) were made by male patients and 17 (48.6\%) by female patients. Mean age was $36.17 \pm 26$ years in the male patients, and $33.56 \pm 14$ years in the female patients. Of the complaint cases, 16 had led to deaths. Other harms leading to complaint are shown in Table 1. All complaints related to anesthetic services provided in hospitals, and none related to anesthesia in doctor's offices or clinics. In total, $62.8 \%$ of events leading to complaints had occurred in the operation room, of which, 5.7\% were before inducing anesthesia and 57.1\% were during induction and maintaining anesthesia.

General anesthesia received $80 \%$ of complaints, spinal anesthesia $14.3 \%$, epidural anesthesia $2.9 \%$, and monitoring anesthesia $2.9 \%$.

Twenty-eight complaint cases (80\%) were for elective surgeries, and 7 cases (20\%) for emergency surgery.

Of the 16 cases leading to death, $11(68.8 \%)$ happened in the operating room, $2(12.5 \%)$ in recovery room, and $3(18.8 \%)$ in the ICU.

\begin{tabular}{|lcc|}
\hline Table 1. Frequency Distribution of Complaints Based on Type of Damage to Patients \\
\hline Damage & Frequency & Percentage \\
\hline Death & 16 & 45.7 \\
\hline Brain damage & 2 & 5.7 \\
\hline Backache & 3 & 8.6 \\
\hline Comma & 1 & 2.9 \\
\hline Failure to control hypertension & 1 & 2.9 \\
\hline Hypotension & 1 & 2.9 \\
\hline Dental damage & 1 & 2.9 \\
\hline Paralysis & 2 & 5.7 \\
\hline Headache and nausea & 3 & 8.6 \\
\hline Shortness of breath & 3 & 8.6 \\
\hline Facial damage & 1 & 2.9 \\
\hline Infection & 1 & 2.9 \\
\hline Total & 35 & 100 \\
\hline
\end{tabular}

The person providing anesthetic services was an anesthesiologist in 32 cases (91.4\%), an anesthetic technician in one case (2.9\%), and an assistant anesthesiologist in two cases (5.7\%).

According to the preliminary judicial council vote, the anesthesiologist was blamed in 21 cases (60\%), and acquitted in 12 cases (34.3\%), and the hearing in the Medical Council was terminated by the complainant's consent in two cases.

In 16 death cases, according to the preliminary judicial council vote, the anesthesiologist was blamed for negligence in 8 cases (50\%) and acquitted in 8 cases (50\%).

The final vote had been issued in $88.6 \%$ of complaint cases, and all confirmed the preliminary sentence, and $11.4 \%$ of cases were still under judicial consideration and the final sentence had not been issued.

In cases leading to deaths, in which, the anesthesiologist negligence had been confirmed by the Medical Judiciary Council, the reason for negligence was choosing the wrong anesthetic method or inadequate preoperative diagnostic measures in three cases, and inadequate care by the anesthesiologist in five cases.

\section{Discussion}

Review of complaints about this study revealed that the majority of complaints were about cases that had led to death, and the anesthesiologist negligence had been confirmed in half of these cases. In addition, 5.7\% of these cases had led to permanent brain damage and 5.7\% had caused neurological paralysis, and mean age of people who had been the subject of these complaints was under 40 years. 
Mean age of the deceased was 41.2 years. The majority of complaints and death cases related to elective surgeries.

Review of similar studies show that most anesthesia complaints related to permanent brain damage and deaths, but complaints in reports and articles about dental, airway, breathing damage, and complaints about neuraxial blocks and awareness were several times those found in the present study, which may be due to several factors, including obtaining consents from the patients and accompaniments concerning possible complications during and after surgery, and also the people's particular attitude toward such complications that makes them refrain from complaining in cases of mild complications. Furthermore, the notion that there will be no authority to determine damage even when the damage is confirmed may be effective in not complaining about mild damage. Generally, the patients' lack of knowledge that they could complain even in cases of mild damage can be an important factor for the low number of complaints, and they are probably satisfied with merely filing their complaint in the hospital (2-5).

In a study conducted by Aitkenhead in America, anesthesia-induced maternal and neonatal mortality rates before 1990 and 1990 - 2003 were compared. Before 1990, death and brain damage were the most cause of complaints, and between 1990 and 2003, backache and peripheral nerve damage constituted the most complaints about midwifery anesthesia (8).

Jimenez et al. studied pediatric anesthesia complaints over three decades from 1973 to 2000 , and found the majority of complaints were about cases that had led to deaths, brain damage, and respiratory events, and factors that accompanied death and brain damage complaints included cardiovascular and respiratory events (9).

Cheney et al. investigated the wrong anesthetic techniques in procedures conducted outside operating room in America. Twenty-four complaints about deaths and permanent brain damage during general anesthesia or anesthetic care outside operation room had been registered, and $58 \%$ of anesthesia outside operating room had been performed by monitored anesthesia care (MAC) technique. Half of these complaints concerned gastrointestinal ward due to inadequate oxygenation and ventilation. The cause of death had been registered as inadequate and substandard care. This study emphasized that anesthesia damage can be severer outside operating room than inside operating room (10).

Given the present study results, and the young age of most cases leading to death and elective surgeries, it can be concluded that greater care during anesthesia, correct choice of anesthetic method, and proper diagnostic procedures before surgery can prevent anesthesia events.
It was thought that the present study could facilitate obtaining knowledge about doctors' risks being accused, as well as frequency of wrong anesthetic treatments in this region, but, given the small number of registered complaints that do not match number of deaths and anesthesia-related complications, the frequency and diversity of damage could not be found. Given the need for registration of complications caused during medical services, it is recommended that morbidity and mortality committees of hospitals register their cases in an integrated system, so that such information can be used for the practical objectives of assessing medical complications.

\subsection{Conclusions}

Mean age of anesthesia-related deaths for which the anesthesiologist was blamed indicate that half of death or brain damage cases can be prevented through greater patient care before and during surgery. To find out mild damage caused by anesthesia, these cases should be actively registered in hospitals' error registration systems.

\section{Footnotes}

Conflict of Interests: It is not declared by the author.

Ethical Approval: This article was an extract from a registered thesis (93199) at Kermanshah University of Medical Sciences. Permission obtained from the Research Council and deputy research and technology of Kermanshah University of Medical Sciences, and the Ethics Committee of Imam Reza Clinical Research Development Unit, coordination with the Medical Council of Kermanshah province.

Funding/Support: It is not declared by the author.

\section{References}

1. Crosby E. Medical malpractice and anesthesiology: Literature review and role of the expert witness. Can J Anaesth. 2007;54(3):227-41. doi: 10.1007/BF03022645. [PubMed: 17331936].

2. Cook TM, Bland L, Mihai R, Scott S. Litigation related to anaesthesia: An analysis of claims against the NHS in England 1995-2007. Anaesthesia. 2009;64(7):706-18. doi: 10.1111/j.1365-2044.2009.05913.x. [PubMed: 19624625].

3. Mahfouzi A, Zamani R. The evaluation of the causes of anesthesiologist's legal pursuits in Tehran Medical Council from 1993 to 2003. Iran J Forensic Med. 2007;13(2):98-101.

4. Staender S, Schaer H, Clergue F, Gerber H, Pasch T, Skarvan K, et al. A Swiss anaesthesiology closed claims analysis: Report of events in the years 1987-2008. Eur J Anaesthesiol. 2011;28(2):85-91. doi: 10.1097/EJA.0b013e3283414fea. [PubMed: 21088588].

5. Hachenberg T, Neu J, Werner S, Wiedemann D, SchaffartzikW. [Quality of anesthesiological expert opinion in medical claims cases]. Anaesthesist. 2012;61(6):497-502. German. doi: 10.1007/s00101-012-2031-3. [PubMed: 22576994]. 
6. Cranshaw J, Gupta KJ, Cook TM. Litigation related to drug errors in anaesthesia: An analysis of claims against the NHS in England 1995-2007. Anaesthesia. 2009;64(12):1317-23. doi: 10.1111/j.13652044.2009.06107.x. [PubMed: 19849678].

7. Samarkandi A. Medico-legal liabilities of anesthesia practice in Saudi Arabia. Middle East J Anaesthesiol. 2006;18(4):693-706. doi: 10.1016/j.bpa.2006.09.006. [PubMed:16749564].

8. Aitkenhead AR. Informing and consenting for anaesthesia. Best Pract Res Clin Anaesthesiol. 2006;20(4):507-24. [PubMed: 17219938].
9. Jimenez N, Posner KL, Cheney FW, Caplan RA, Lee LA, Domino KB. An update on pediatric anesthesia liability: A closed claims analysis. Anesth Analg. 2007;104(1):147-53. doi: 10.1213/01.ane.0000246813.04771.03. [PubMed: 17179260].

10. Cheney FW, Posner KL, Lee LA, Caplan RA, Domino KB. Trends in anesthesia-related death and brain damage: A closed claims analysis. Anesthesiology. 2006;105(6):1081-6. doi: 10.1097/00000542200612000-00007. [PubMed: 17122570]. 